\title{
A systematic review and meta-analysis of early diagnosis and treatment of hypertensive stroke under calcium channel blockers
}

\author{
Aiping Feng ${ }^{1}$, Wen Wang ${ }^{1}$, Chen $\mathrm{Du}^{1}$, Mingjie $\mathrm{He}^{2}$ \\ ${ }^{1}$ Department of Neurosurgery, Deyang Second People's Hospital, Deyang, China; ${ }^{2}$ Department of Neurosurgery, The First Affiliated Hospital of \\ Chengdu Medical College, Chengdu, China \\ Contributions: (I) Conception and design: A Fen, M He; (II) Administrative support: W Wang; (III) Provision of study materials or patients: A Fen, W \\ Wang, C Du; (IV) Collection and assembly of data: All authors; (V) Data analysis and interpretation: A Fen, C Du, M He; (VI) Manuscript writing: \\ All authors; (VII) Final approval of manuscript: All authors. \\ Correspondence to: Mingjie He. Department of Neurosurgery, The First Affiliated Hospital of Chengdu Medical College, Chengdu, China. \\ Email: hesjlove00@163.com.
}

\begin{abstract}
Background A systematic evaluation of the therapeutic effects of calcium channel blockers (CCB) on stroke is carried out to provide clinical evidence for their application.

Methods: A search for randomized controlled trials (RCTs) of CCB in the treatment of stroke patients in the electronic databases of PubMed, Embase, Medline, Spring, and Ovid from their establishment to January 31, 2021 was performed, and the collected studies were then screened for the exclusion criteria. The Cochrane Handbook version 5.0.2 system evaluation writing manual was adopted to evaluate the risk of bias for the included literature, and a meta-analysis using Review Manager 5.3 software was applied.
\end{abstract}

Results: A total of 13 RCTs were included, comprising 1,067 subjects. The meta-analysis showed that the recurrence rate of stroke in patients from the observation group reduced sharply after CCB treatment [mean difference $(M D)=0.41,95 \%$ confidence interval $(C I): 0.24-0.70, Z=3.31, P=0.0009]$, the Mini-Mental State Examination (MMSE) score improved markedly ( $M D=2.82,95 \% \mathrm{CI}: 1.69-3.95, \mathrm{Z}=4.89, \mathrm{P}<0.00001$ ), and the Montreal Cognitive Assessment (MoCA) score was obviously increased (MD =6.07, 95\% CI: 0.34-11.81, $\mathrm{Z}=2.08, \mathrm{P}=0.04)$. Moreover, the diastolic blood pressure of the observation group decreased steeply after CCB treatment $(M D=-1.11,95 \% \mathrm{CI}:-2.06-0.15, \mathrm{Z}=2.27$, and $\mathrm{P}=0.02$ ). However, the effective rate of clinical treatment did not increase hugely ( $\mathrm{MD}=1.70,95 \% \mathrm{CI}: 0.50-5.83, \mathrm{Z}=0.85, \mathrm{P}=0.40)$, and systolic blood pressure did not drop sharply ( $M D=-1.24,95 \% \mathrm{CI}:-2.85-0.37, \mathrm{Z}=1.51, \mathrm{P}=0.13$ ).

Discussion: CCB treatment of stroke effectively prevented stroke recurrence, and showed faster recovery of cognitive function, and better lowering of blood pressure, all of which makes CCBs suitable for the treatment of stroke.

Keywords: Blood pressure; calcium channel blockers; meta-analysis; stroke

Submitted Apr 16, 2021. Accepted for publication Jun 11, 2021.

doi: 10.21037/apm-21-1272

View this article at: https://dx.doi.org/10.21037/apm-21-1272

\section{Introduction}

With societal changes and aging of populations, the incidence of stroke is increasing. It has become the world's first disability factor and the second leading cause of death (1). Essentially, the cause of stroke is the rupture, stenosis or occlusion of the intracranial arteries through external factors. The incidence, disability, recurrence, and mortality rate of stroke are high, with survivors suffering from a certain degree of cognitive impairment, dementia, paralysis, and other sequelae, which place a heavy psychological burden on both patients and their family members (2). Scientific research shows that hypertension is one of the important causes of stroke, and it is also the focus 
of the management and prevention of stroke. There are two types of stroke: hemorrhagic and ischemic. Hemorrhagic stroke is caused by the rupture of the cerebral artery supplying blood to the brain tissue, and the causative factors of ischemic stroke are stenosis or occlusion of the cerebral artery supplying blood. Furthermore, the main independent risk factor for both types of stroke is hypertension (3). Therefore, actively controlling patients' blood pressure is key to preventing stroke.

Current antihypertensive drugs can be divided into 5 types: calcium channel blockers (CCB), angiotensin converting enzyme inhibitors, diuretics, angiotensin II receptor blockers, and $\beta$-receptor blockers (4). The main mechanism of action of CCBs to reduce blood pressure is competition with calcium ions in the calcium ion channel located on vascular smooth muscle, inhibiting calcium ions from entering into cells, interfering with the contraction process of cells, and expanding vascular smooth muscle (5). So far, CCB has achieved certain results in the antihypertensive treatment of stroke patients. Many clinical randomized controlled trials (RCTs) have shown that $\mathrm{CCB}$ has a good effect on reducing blood pressure and incidence of stroke in patients with stroke, and protecting blood vessels in patients with stroke. However, there are many types of CCBs, and no systematic reviews on stroke prevention and treatment. In addition, the quality of the literature is uneven, so it is difficult to obtain unified clinical treatment evidence. Therefore, this study conducted a meta-analysis of related RCTs of CCB in the treatment of hypertensive stroke to provide the clinical basis for the application of CCB in the prevention and treatment of stroke.

We present the following article in accordance with the PRISMA reporting checklist (available at https://dx.doi. org/10.21037/apm-21-1272).

\section{Methods}

\section{Literature inclusion and exclusion criteria}

The criteria for inclusion were defined as follows. First, publicly available RCTs in English on the CCB treatment of hypertensive stroke published from the establishment of English-language databases to January 31, 2021. Second, the research subjects were clinically diagnosed as stroke [diastolic blood pressure (DBP) $\geq 90 \mathrm{mmHg}$ and systolic blood pressure $(\mathrm{SBP}) \geq 140 \mathrm{mmHg}$, and at least 18 years old, regardless of sex. Third, an experimental group and a control group were defined: the intervention measure of the experimental group was CCB therapy only (including amlodipine, nifedipine, and nimodipine), and patients in this group could not be treated with combination treatments; the control group received non-CCB treatment. Fourth, the type of stroke was not limited. Fifth, the baseline data of patients were comparable.

The criteria for exclusion included the following. First, the type of research was retrospective, case review, or other non-RCT research. Second, the literatures whose language were not English. Third, the research subjects were animals or tissue samples. Fourth, the research subjects were nonstroke patients, or patients with stroke combined with other diseases. Fifth, CCB combination treatment of stroke in the experimental group, or CCB treatment of the control group. Sixth, research results were not reported, or incomplete research data led to failure to calculate the corresponding indicators.

\section{Literature search}

Five English-language electronic databases (PubMed, Embase, Medline, Spring, and Ovid) were electronically searched using search terms comprising subject terms and free terms: "hypertension", "stroke", "cerebral apoplexy", "cerebral infarction", "calcium channel blockers", "calcium antagonist", "CCB", "Nimodipine", "Amlodipin”, and "Nifedipine". The literature search was done by two researchers (Feng and Wang) in a back-to-back manner.

\section{Literature screening}

The literature screening was completed independently by two researchers (Feng and Wang). After the document search, the citations were first imported into NoteExpress3.2 software to generate a literature database, and NoteExpress 3.2 software was used to check for duplicates, which were removed. By browsing document title and abstract, clearly unrelated literature was removed. Finally, the full text of each study was carefully read, and strictly screened according to the inclusion and exclusion criteria to determine inclusion. If the data description was unclear, we attempted to contact the respective author for detailed information. If no reply was received, the study was rejected. In the literature screening process, the two researchers discussed and resolved or reached a consensus after consultation with a third researcher. 


\section{Data extraction}

When extracting the data, two researchers first extracted it independently, it was entered into an Excel table, and there was cross-checking after extraction was completed. If there were differences in opinions, it was resolved by discussion or consultation with a third researcher. The extracted content included basic information of the study (document title, first author, publication year, and published journal), basic characteristics of the research subjects (sex, age, disease course, research sample size, and baseline comparability), research design, intervention measures of the experimental group and the control group, research evaluation indicators, and research data.

\section{Evaluation criteria for literature bias}

The literature bias risk assessment was completed using the Cochrane Handbook version 5.0.2 systematic review writing manual. The quality evaluation has seven aspects. First is random sequence generation, and whether the research subjects were randomly grouped using a random number table or other randomization methods. Second is allocation concealment, and whether random number tables or other random methods were used for grouping and confidentiality. Third is subject blinding, and whether the clinical research objects were aware of their participation in the study, their group, and the interventions they received. Fourth is blindness of the outcome assessors, and whether outcome assessors know the group of the subjects and the interventions they received. Fifth is data completeness, and whether the research data were complete and whether there were any missing circumstances. Sixth is selective reporting, and whether there was selective reporting in the research. Seventh is other biases. In the bias evaluation and analysis of the included studies, the two researchers discussed and resolved disagreements in opinion or the third researcher would arbitrate if necessary.

\section{Statistical methods}

The Cochrane Handbook version 5.0.2 system review writing manual was used to evaluate the risk of bias for the included studies, Review Manager5.3 software was used to conduct a meta-analysis of the literature, and forest and funnel diagrams were output based on the analysis results. In meta-analysis, the RCTs were tested for heterogeneity, and the $\mathrm{I}^{2}$ test was adopted to evaluate the heterogeneity of the included studies. If the result of the heterogeneity test was $\mathrm{I}^{2} \geq 50$ and $\mathrm{P}<0.05$, it indicated homogeneity among the included studies. The fixed effect model (FEM) was used for the meta-analysis of the combined effect size. If the heterogeneity test result was $\mathrm{I}^{2}<50$ and $\mathrm{P}>0.05$, it indicated heterogeneity among the included studies, and the metaanalysis used the random effect model (REM). The effective rate of clinical treatment, the recurrence rate of stroke after treatment, and other binary variables used relative risk as the effect size, and the $95 \%$ confidence interval (CI) was calculated. For continuous variables such as Mini-Mental State Examination (MMSE) score, Montreal Cognitive Assessment (MoCA) score, and BP after treatment, the weighted mean difference was used as the effect size. If the units of the indicators were different, the standardized MD (SMD) was used as the effect size to calculate the 95\% CI. The u test ( $\mathrm{Z}$ test) was adopted to judge whether there was statistical significance between the combined statistics, the probability $P$ value was measured according to the u value, and $\alpha=0.05$ was used as the test level. If $\mathrm{P}<0.05$, there was statistical significance of the combined statistic. For binary variables, if $95 \% \mathrm{CI}$ did not contain 1 (i.e., $95 \% \mathrm{CI}>1$ or $95 \% \mathrm{CI}<1$ ), it was equivalent to $\mathrm{P}<0.05$, which meant that the combined statistic was statistically substantial. For continuous variables, if $95 \% \mathrm{CI}$ did not contain 0 (i.e., $95 \%$ $\mathrm{CI}>0$ or $95 \% \mathrm{CI}<0$ ), it was equivalent to $\mathrm{P}<0.05$, which meant the combined statistic was statistically marked.

\section{Results}

\section{Literature search results}

The preliminary search for RCTs on the treatment of hypertensive stroke with calcium antagonists published up to January 31, 2021 revealed 994 related studies (381 articles from PubMed, 109 from Embase, 142 from Medline, 205 from Spring, and 157 from Ovid). After elimination of duplicate documents, the title and abstract of 802 articles were read and after irrelevant documents were excluded, 126 articles remained. Finally, a total of 13 articles (6-18) were included after careful reading and strict screening based on the inclusion and exclusion criteria. The 13 articles were all publicly available RCTs, published from 1988 to 2019, and contained 1,067 research subjects, including 527 in the experimental group and 540 in the control group. The baseline data of the two groups were comparable. A flow chart of document search is shown in Figure 1, and the basic literature information is shown in Table 1 . 


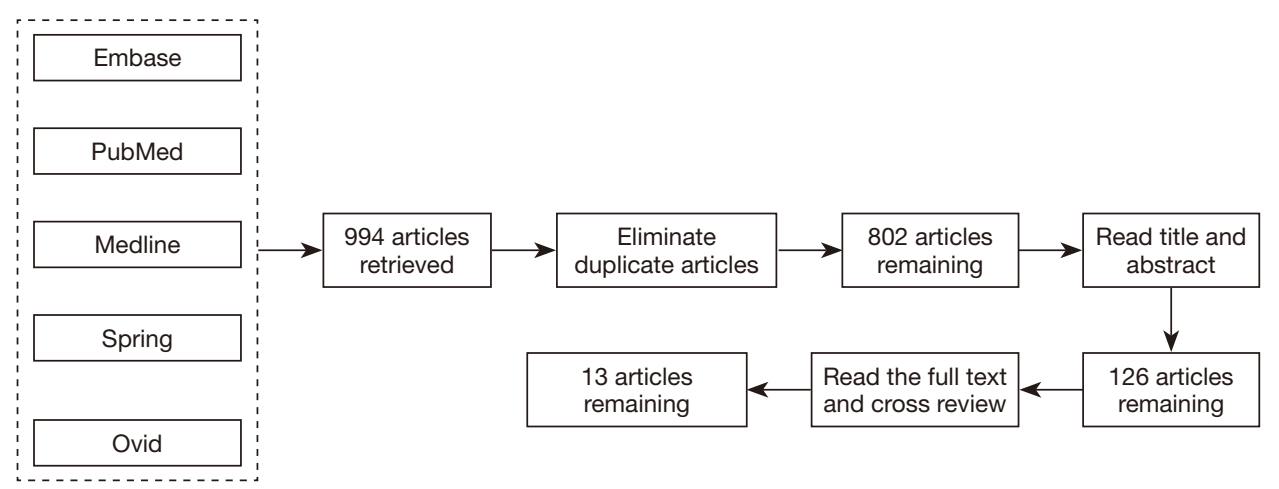

Figure 1 Flow chart of document search.

\section{Bias risk assessment}

Based on the seven aspects of risk bias in the Cochrane Handbook 5.0.2 the evaluation of bias risk in this study included the following: (I) random sequence generation. The 13 studies included in this study all reported random grouping. The study of Lin et al. clearly indicated that the random method was a random number table, suggesting low risk. The study by Pagan et al. only mentioned random grouping, but did not tell which random method was used, suggesting unclear risk. (II) Allocation concealment. The study of Fagan et al. mentioned that the allocation of subjects was hidden, indicating that it was low risk. The study of Liang et al. did not mention whether to allocate concealment, indicating that the risk was unclear. (III) Subjects blinding. The study by Fagan et al. mentioned that "patients understood this study and signed the informed consent form", indicating that it was high risk. Liang et al.'s study did not mention whether to blind the subjects, suggesting that the risk was unclear. (IV) Blindness of outcome evaluators. The study by Fagan et al. mentioned that there was blinding of outcome evaluators, suggesting low risk. Among the other nine articles, Liang et al.'s study did not mention whether to blind the outcome assessor, suggesting that the risk was unclear. (V) Data integrity. All 13 literatures had complete data, suggesting low risk. (VI) Selective report. There was no selective report in 13 literatures after analysis, indicating low risk. (VII) Other biases. The number of patients in the experimental group and the control group in the study of Fagan et al. was different, suggesting a high risk. Whether there were other biases in study of Liang et al. can't be determined, suggesting that the risk was unclear. The results of this bias risk assessment are shown in Figures 2 and 3.

\section{Effective rate of clinical treatment}

A total of 5 of the 13 RCTs included in this study reported on the effective rate of CCB therapy in the treatment of hypertensive stroke. A total of 336 stroke patients were included, of which 164 were in the experimental group (135 cases were effective) and 172 cases were in the control group (124 cases were effective). The heterogeneity test found a certain degree of heterogeneity among the 5 included studies $\left(\mathrm{I}^{2}=75 \%\right.$ and $\left.\mathrm{P}=0.003\right)$, so the REM was used for analysis, and the results are shown in Figure 4. The combined effect of the metaanalysis was $\mathrm{MD}=1.70,95 \% \mathrm{CI}: 0.50-5.83, \mathrm{Z}=0.85$, and $\mathrm{P}=0.40$, and the difference was not statistically substantial, which showed that CCB treatment for hypertensive stroke had no marked clinical effect compared with other treatments.

\section{Recurrence rate of stroke}

A total of 4 of the 13 documents included in this study reported on the recurrence rate of stroke after CCB treatment of hypertensive stroke. A total of 423 stroke patients were included, and there were 212 cases in the experimental group (25 with recurrence after treatment) and 211 cases in the control group (51 with recurrence after treatment). The heterogeneity test found that the 4 included studies had good homogeneity $\left(\mathrm{I}^{2}=4 \%\right.$ and $\mathrm{P}=0.37$ ), so the FEM was used for analysis, and the results are presented in Figure 5. The combined effect of the meta-analysis ( $\mathrm{MD}=0.41,95 \% \mathrm{CI}: 0.24-0.70, \mathrm{Z}=3.31$, and $\mathrm{P}=0.0009)$ showed a statistically obvious difference, which indicated that CCB could reduce the recurrence rate of post-treatment stroke. 
Table 1 Basic literature information of the included studies

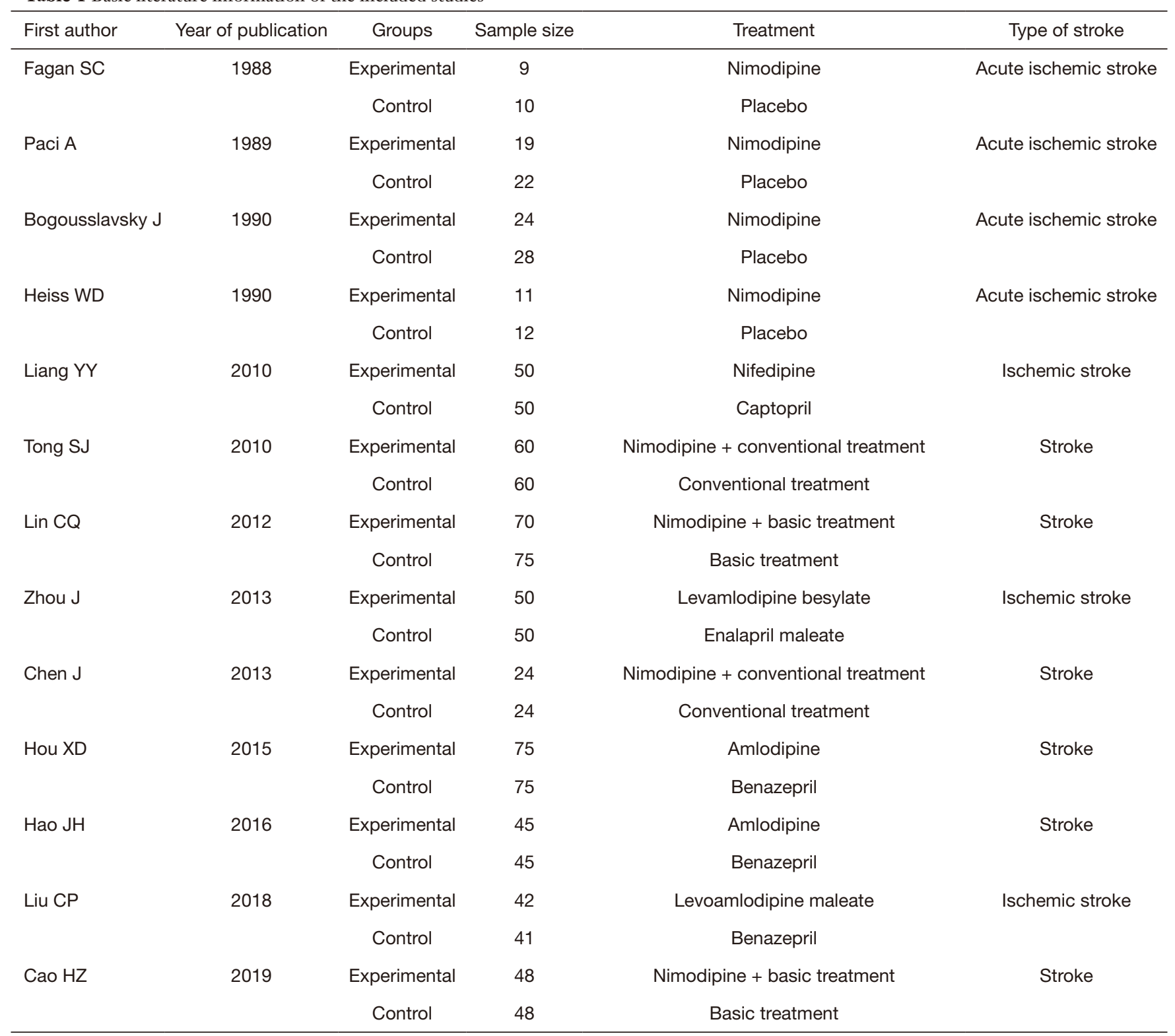

\section{MMSE scoring}

Among the 13 articles included in this study, a total of 3 reported on the MMSE scores of CCB in the treatment of hypertensive stroke. A total of 264 stroke patients were included, of whom 132 were in the experimental group and 132 were in the control group. The heterogeneity test revealed that the 3 included studies had good homogeneity $\left(\mathrm{I}^{2}=0 \%\right.$ and $\left.\mathrm{P}=0.45\right)$, so the FEM was used for analysis, and the results are displayed in Figure 6. The combined effect of the meta-analysis (MD $=2.82$; 95\% CI: 1.69-3.95,
$\mathrm{Z}=4.89$, and $\mathrm{P}<0.00001)$ had a statistically great difference, indicating that stroke patients treated with CCB had higher MMSE scores compared with other treatments.

\section{MoCA scoring}

A total of 3 of the 13 documents included in this study reported on the MoCA scores of CCB after hypertensive stroke. A total of 289 stroke patients were included, of whom 142 were in the experimental group and 147 were in 


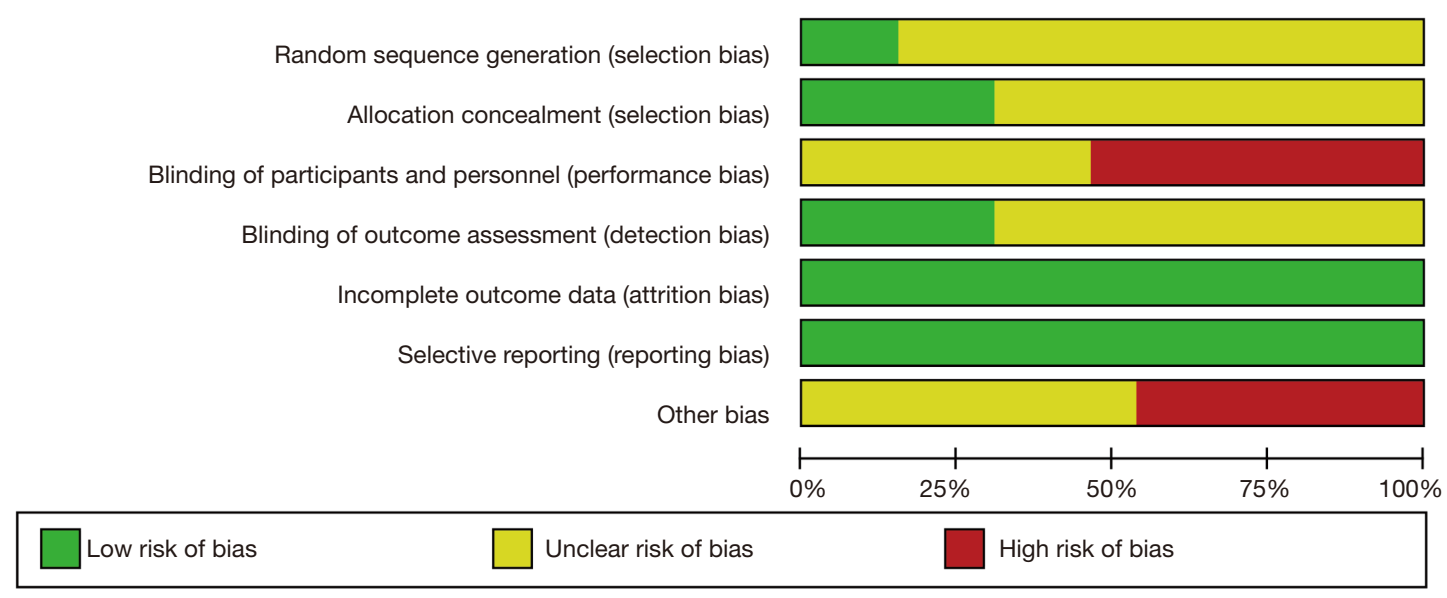

Figure 2 Bar chart of risk bias risk analysis of included studies.

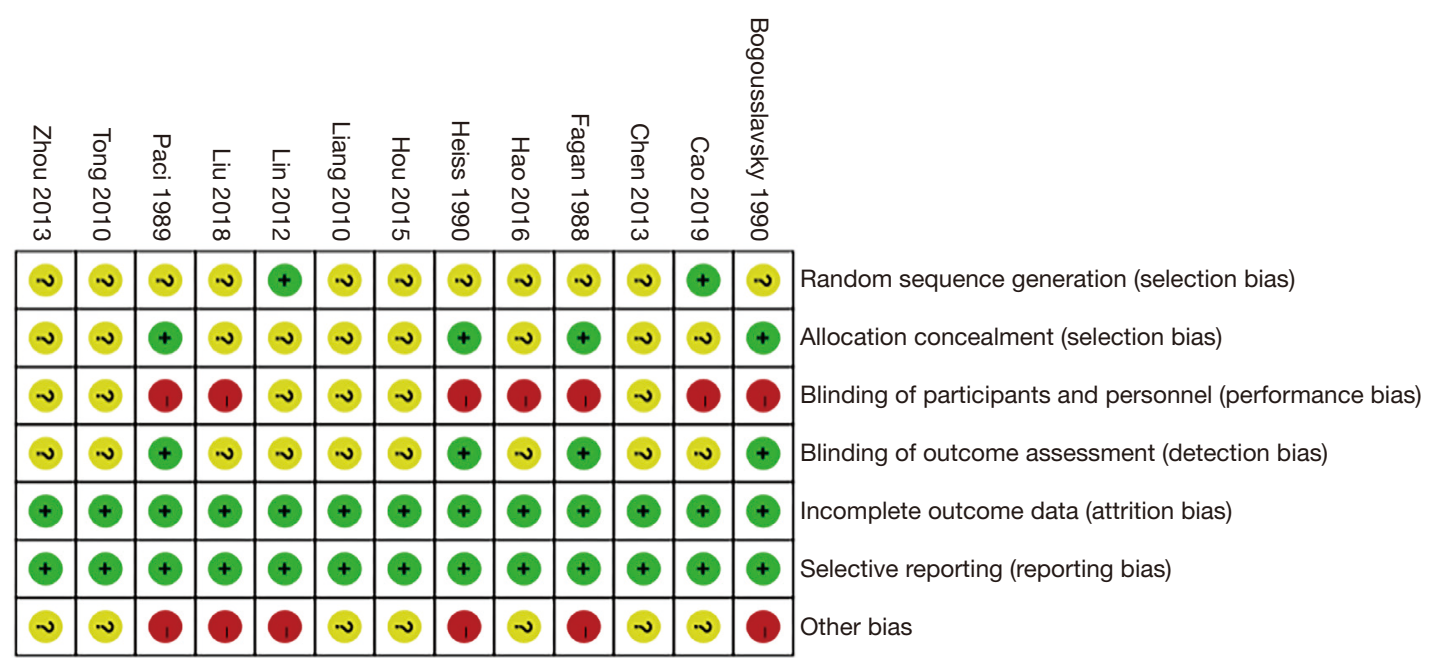

Figure 3 Analysis of the risk of bias in the included studies.

\begin{tabular}{|c|c|c|c|c|c|c|c|c|c|c|}
\hline \multirow[b]{2}{*}{ Study or Subgroup } & \multicolumn{2}{|c|}{ Experimental } & \multicolumn{2}{|c|}{ Control } & \multirow{2}{*}{ Weight } & \multirow{2}{*}{$\begin{array}{c}\text { Odds Ratio } \\
\text { M-H, Random, } 95 \% \mathrm{Cl}\end{array}$} & \multirow{2}{*}{\multicolumn{3}{|c|}{$\begin{array}{c}\text { Odds Ratio } \\
\text { M-H, Random, } 95 \% \mathrm{Cl}\end{array}$}} & \\
\hline & Events & Total & Events & Total & & & & & & \\
\hline Bogousslavsky 1990 & 18 & 24 & 24 & 28 & $20.7 \%$ & $0.50[0.12,2.04]$ & & $\longrightarrow$ & & \\
\hline Heiss 1990 & 9 & 11 & 9 & 12 & $16.1 \%$ & $1.50[0.20,11.24]$ & & & & \\
\hline Liang 2010 & 38 & 50 & 43 & 50 & $23.6 \%$ & $0.52[0.18,1.44]$ & & & & \\
\hline Paci 1989 & 18 & 19 & 12 & 22 & $15.0 \%$ & $15.00[1.69,132.90]$ & & & & \\
\hline Tong 2010 & 52 & 60 & 36 & 60 & $24.5 \%$ & $4.33[1.75,10.72]$ & & & 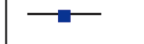 & \\
\hline Total $(95 \% \mathrm{Cl})$ & & 164 & & 172 & $100.0 \%$ & $1.70[0.50,5.83]$ & & & & \\
\hline Total events & 135 & & 124 & & & & & & & \\
\hline $\begin{array}{l}\text { Heterogeneity: } \mathrm{Tau}^{2}= \\
\text { Test for overall effect: }\end{array}$ & $\begin{array}{l}.40 ; \mathrm{Chi}^{2}= \\
=0.85(\mathrm{P}\end{array}$ & $\begin{array}{l}16.07, \\
=0.40)\end{array}$ & $\mathrm{df}=4(\mathrm{P}$ & $=0.003$ & $3) ; 1^{2}=75^{\circ}$ & & 0.001 & $\begin{array}{l}0.1 \\
\text { perimental] }\end{array}$ & $\begin{array}{r}1 \\
\text { [control] }\end{array}$ & 1000 \\
\hline
\end{tabular}

Figure 4 Forest diagram of clinical treatment effective rate of patients from the experimental and control groups. CI, confidence interval. 


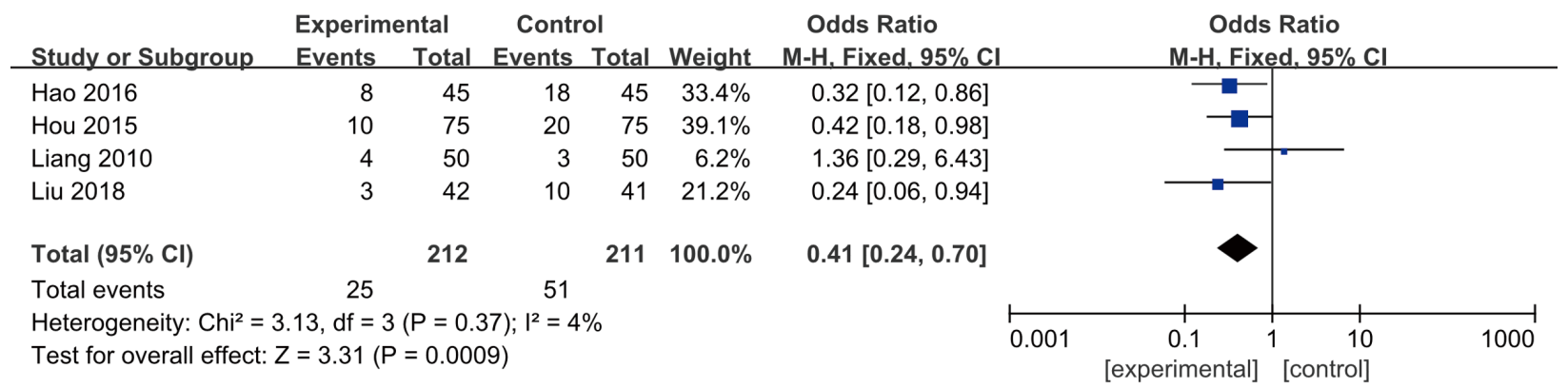

Figure 5 Forest map of stroke recurrence rate after treatment in patients from the experimental and control groups. CI, confidence interval.

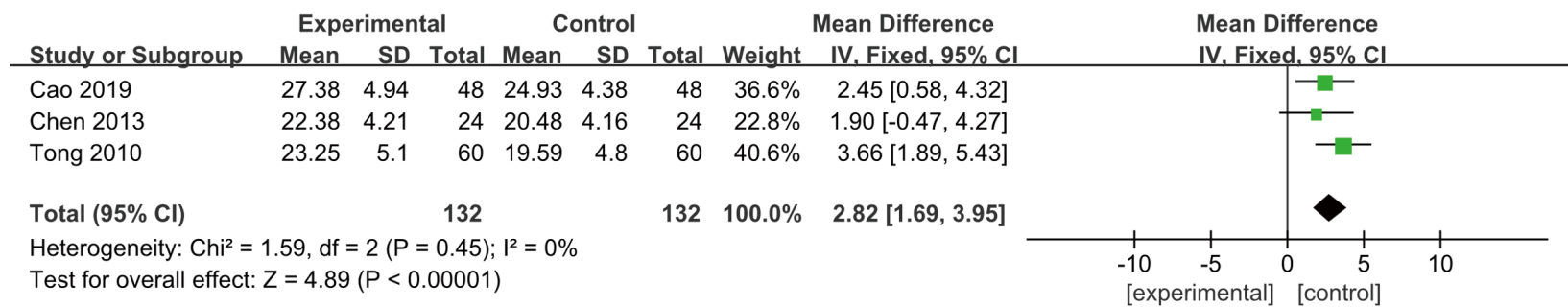

Figure 6 Forest map of Mini-Mental State Examination scores after treatment of patients from the experimental and control groups. CI, confidence interval.

the control group. The heterogeneity test showed a certain degree of heterogeneity among the 3 included studies $\left(\mathrm{I}^{2}=96 \%\right.$ and $\mathrm{P}<0.00001)$, so the REM was used for analysis, and the results are presented in Figure 7. The combined effect of the meta-analysis $(\mathrm{MD}=6.07 ; 95 \% \mathrm{CI}: 0.34-11.81 ; \mathrm{Z}=2.08$; and $\mathrm{P}=0.04$ ) had a statistically huge difference, which indicated that the MoCA scores of stroke patients treated with CCB were higher than the scores of other treatments.

\section{Systolic blood pressure}

A total of 7 of the 13 articles included in this study reported on SBP after CCB in the treatment of hypertensive stroke, and 535 stroke patients were included: 264 in the experimental group and 271 in the control group. The heterogeneity test indicated that the 7 included studies had good homogeneity $\left(\mathrm{I}^{2}=0 \%\right.$ and $\left.\mathrm{P}=0.71\right)$, so the $\mathrm{FEM}$ was used for analysis, and the results are shown in Figure 8 . The combined effect of the meta-analysis ( $\mathrm{MD}=-1.24 ; 95 \% \mathrm{CI}$ : -2.85-0.37; $Z=1.51 ; \mathrm{P}=0.13)$ showed that the difference was not statistically remarkable, suggesting there was not a significant decrease in SBP after stroke treated with CCB compared with other treatments.

\section{Diastolic blood pressure}

A total of 5 of the 13 articles included in this study reported on DBP after CCB treatment for hypertensive stroke. A total of 416 stroke patients were included, of which the experimental group had 205 and the control group had 211. The heterogeneity test found that the 5 included studies had good homogeneity $\left(\mathrm{I}^{2}=0 \%\right.$ and $\left.\mathrm{P}=0.97\right)$, so the FEM was used for analysis, and the results are shown in Figure 9. The combined effect of meta-analysis (MD $=-1.11,95 \%$ CI: $-2.06-0.15, Z=2.27, P=0.02$ ) showed a statistically huge difference, which indicated that CCBs reduced DBP more significantly after stroke compared with other treatments.

\section{Analysis of publication bias}

The Review Manager 5.3 software was used to construct funnel charts of each outcome indicator of CCB treatment of stroke and perform the publication bias analysis. The results are shown in Figure 10. Some representative documents in the funnel chart of MoCA scoring after patient treatment fell outside the CI, which suggested a certain publication bias in the included literature. In the funnel charts of patients' clinical treatment effective rate, 


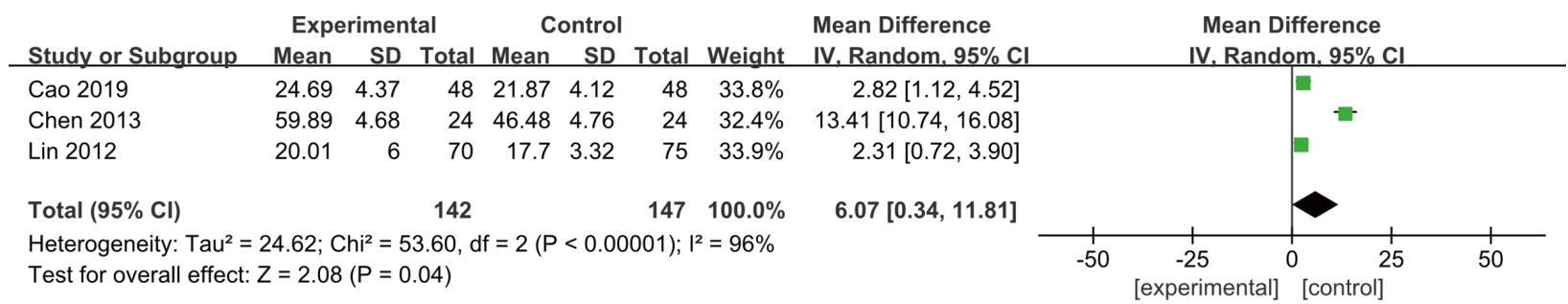

Figure 7 Forest map of Montreal Cognitive Assessment scores after treatment of patients from the experimental and control groups. CI, confidence interval.

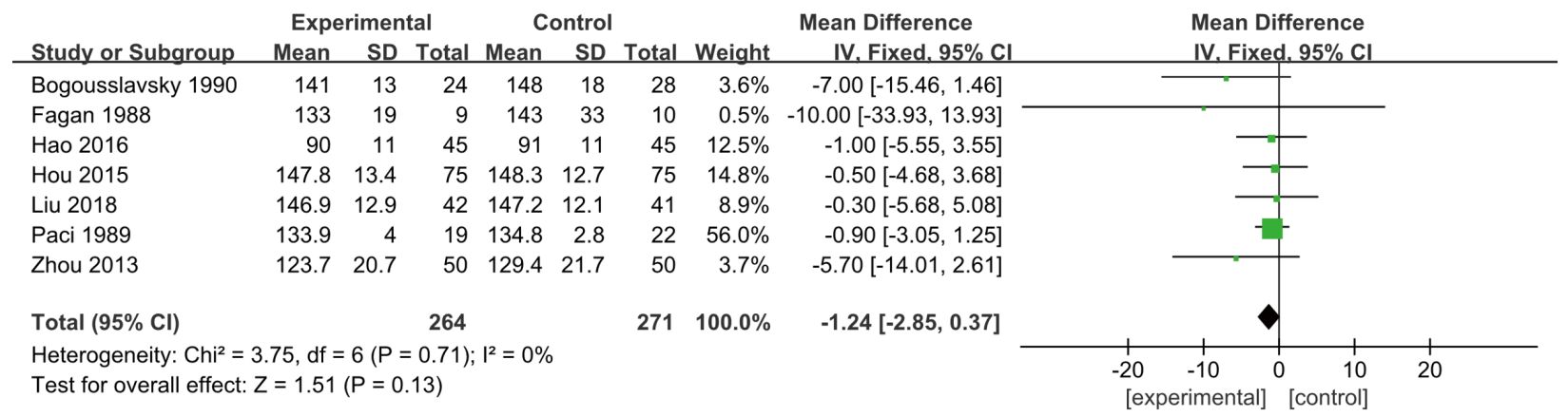

Figure 8 Forest map of systolic blood pressure after treatment in patients from the experimental and control groups. CI, confidence interval.

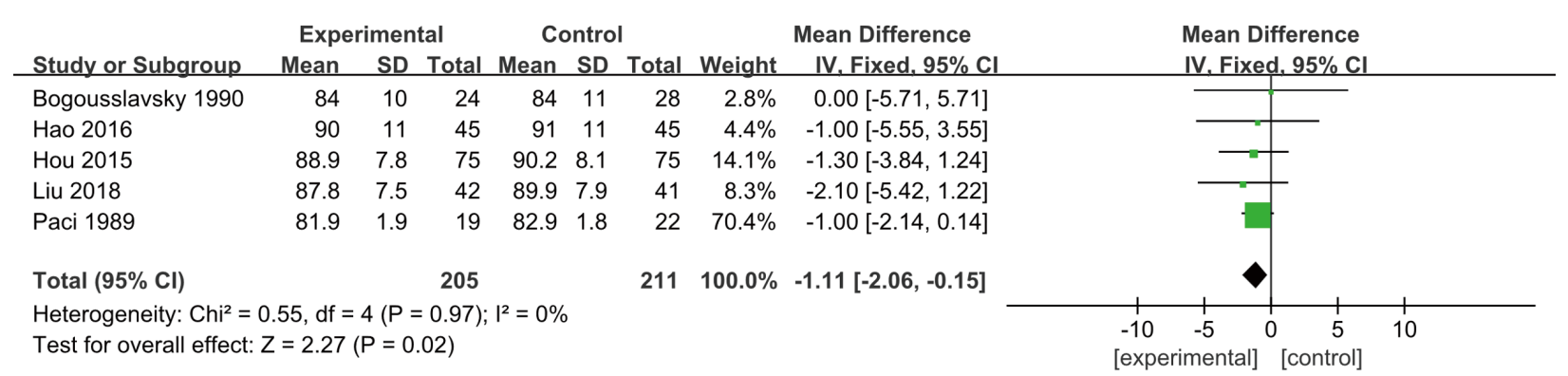

Figure 9 Forest map of diastolic blood pressure after treatment in patients from the experimental and control groups. CI, confidence interval.

stroke recurrence rate, MMSE score, SBP, and DBP, the scattered points were essentially distributed within the CI, showing that the possibility of literature publication bias was small.

\section{Discussion}

In recent years, the incidence of stroke has risen, with the increasing number of patients with hypertension. The disability and fatality rates of stroke are both high. In
China, $\approx 1.6$ million people die from stroke each year (19), and elevated BP is a basic clinical feature of stroke patients. Therefore, BP management is critical. Treatment of acute stroke patients is usually ineffective, because the brain tissue has been irreversibly damaged, and so all treatments are aimed at improving the defective local brain function (20). When a stroke occurs, a large amount of oxygen free radicals is produced. The increased metabolism of nerve cells causes a large loss of calcium ions, and the overload of calcium ions in the cells leads to the release of a large 

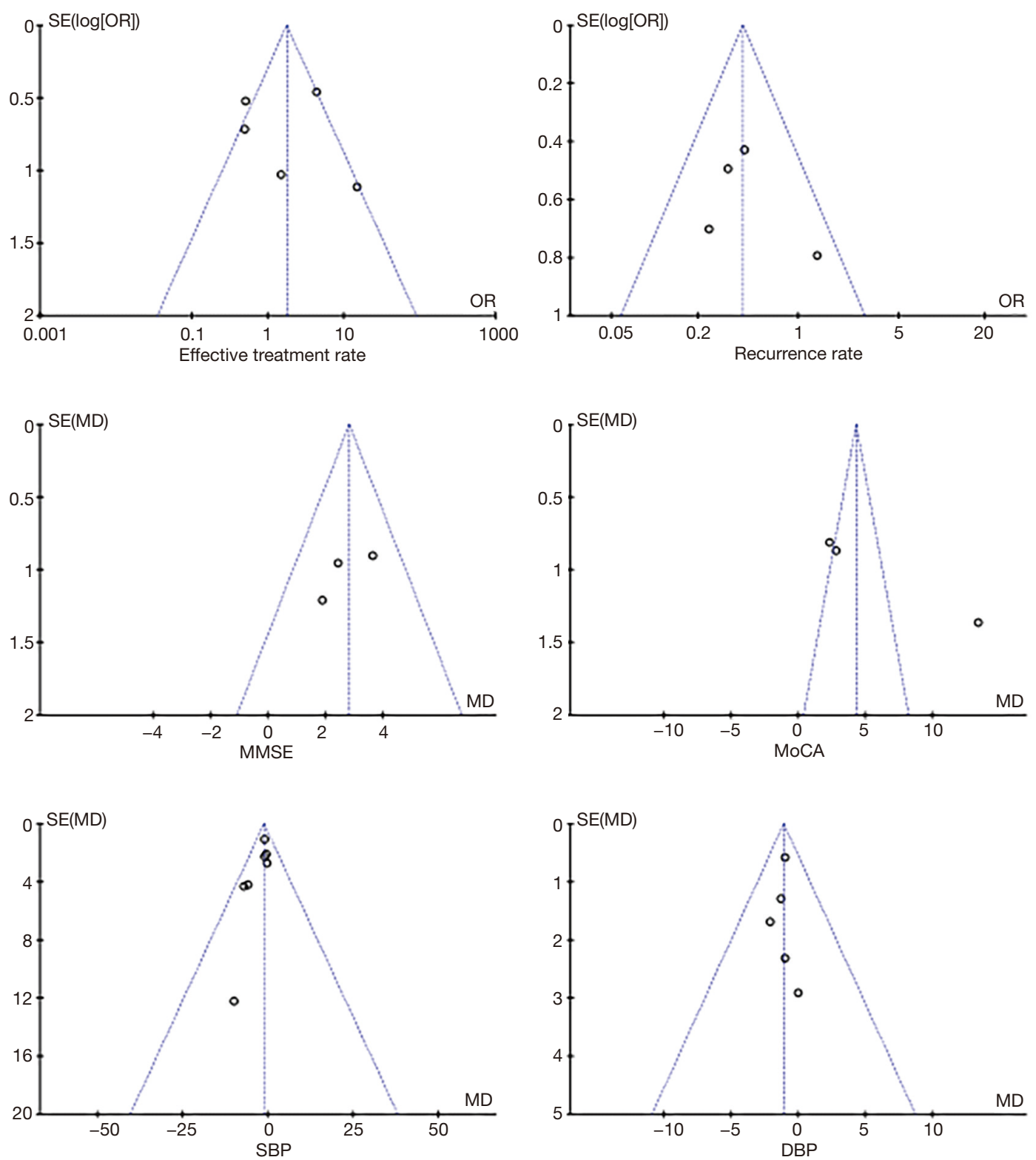

Figure 10 Funnel diagrams of various evaluation indicators. DBP, diastolic blood pressure; MMSE, Mini-Mental State Examination; MoCA, Montreal Cognitive Assessment; SBP, systolic blood pressure.

number of excitatory amino acids, thereby causing calcium ion deposition in cell mitochondria, necrosis of nerve cells, and deepening of brain damage. Scientific research has found that calcium ion overload is the pathway of cell death after stroke (21), so the mechanism of CCBs makes them applicable in the treatment of stroke.

For our meta-analysis of the effect of CCBs in the treatment of stroke, a total of 13 articles were included, comprising 1,067 research subjects, and we assessed the effective rate of clinical treatment, stroke recurrence rate, MMSE score, and MoCA of stroke patients after treatment with CCB. The MMSE score was employed to evaluate the cognitive function of patients after treatment, and the higher the score, the better the cognitive ability. The MoCA score was adopted to evaluate the daily living ability of patients after treatment, and a higher score indicated better daily living ability of the patient. The meta-analysis revealed that compared with other treatments, the clinical treatment 
effect of CCBs for stroke was not marked, and the patients' SBP was not greatly reduced. However, CCB treatment could obviously reduce the recurrence rate of stroke, the DBP of patients was decreased more significantly, and the MMSE and MoCA scores were higher, which indicated that CCB could help stroke patients' cognitive function recovery.

\section{Conclusions}

Our analysis of RCT results for current CCBs in the treatment of stroke found that CCBs can effectively prevent the recurrence of stroke, more quickly recover cognitive function, and lower blood pressure better. However, there are still some shortcomings to this study, which is reflected in the fact that there were few studies included, and the sample size of some RCTs was small, resulting in some results not being completely convincing. Therefore, more high-quality, large-sample, and multicenter clinical RCTs are still needed to confirm the clinical efficacy of CCBs in the treatment of stroke.

\section{Acknowledgments}

Funding: None.

\section{Footnote}

Reporting Checklist: The authors have completed the PRISMA reporting checklist. Available at https://dx.doi. org/10.21037/apm-21-1272

Conflicts of Interest: All authors have completed the ICMJE uniform disclosure form (available at https://dx.doi. org/10.21037/apm-21-1272). The authors have no conflicts of interest to declare.

Ethical Statement: The authors are accountable for all aspects of the work in ensuring that questions related to the accuracy or integrity of any part of the work are appropriately investigated and resolved.

Open Access Statement: This is an Open Access article distributed in accordance with the Creative Commons Attribution-NonCommercial-NoDerivs 4.0 International License (CC BY-NC-ND 4.0), which permits the noncommercial replication and distribution of the article with the strict proviso that no changes or edits are made and the original work is properly cited (including links to both the formal publication through the relevant DOI and the license). See: https://creativecommons.org/licenses/by-nc-nd/4.0/.

\section{References}

1. Esenwa C, Gutierrez J. Secondary stroke prevention: challenges and solutions. Vasc Health Risk Manag 2015;11:437-50.

2. Guzik A, Bushnell C. Stroke Epidemiology and Risk Factor Management. Continuum (Minneap Minn) 2017;23:15-39.

3. Pistoia F, Sacco S, Degan D, et al. Hypertension and Stroke: Epidemiological Aspects and Clinical Evaluation. High Blood Press Cardiovasc Prev 2016;23:9-18.

4. Appleton JP, Sprigg N, Bath PM. Blood pressure management in acute stroke. Stroke Vasc Neurol 2016;1:72-82.

5. Papadopoulos DP, Papademetriou V. Aggressive blood pressure control and stroke prevention: role of calcium channel blockers. J Hypertens 2008;26:844-52.

6. agan SC, Gengo FM, Bates V, et al. Effect of nimodipine on blood pressure in acute ischemic stroke in humans. Stroke 1988;19:401-2.

7. Paci A, Ottaviano P, Trenta A, et al. Nimodipine in acute ischemic stroke: a double-blind controlled study. Acta Neurol Scand 1989;80:282-6.

8. Bogousslavsky J, Regli F, Zumstein V, et al. Double-blind study of nimodipine in non-severe stroke. Eur Neurol 1990;30:23-6.

9. Heiss WD, Holthoff V, Pawlik G, et al. Effect of nimodipine on regional cerebral glucose metabolism in patients with acute ischemic stroke as measured by positron emission tomography. J Cereb Blood Flow Metab 1990;10:127-32.

10. Liang YY, Wang XL, Liu HW, et al. Clinical Study of Longze Tang in Controlling Hypertension in Patients with Ischemic Stroke. Hebei Yixue 2010;16:284-6.

11. Tong SJ. Clinical Observation of Nimodipine for 60 Cases of Cerebral Infarction. Zhongguo Yaofang 2010;21:3019-21.

12. Lin CQ, Liu MF, Wang WF. The Efficacy Observation of Nimodipine on Cognitive Dysfunction in Patients with Post-Stroke. Chinese Journal of General Practice 2012;10:863-4.

13. Zhou J. L benzene sulfonic acid amlodipine and enalaprilat in elderly patients with ischemic stroke compared analysis of blood pressure regulation. China Journal of 
Pharmaceutical Economics 2013;(3):195-7.

14. Chen J, Li ZY, Xu H, et al. Effect of nimodipine on cerebral vascular cognitive impairment after stroke prevention and treatment. Anhui Yiyao 2013;34:209-11.

15. Hou XD. Compare the efficacy and recurrence rate of amlodipine and benazeprilthe in the treatment of hypertensive stroke. Journal Of China Prescription Drug 2015;13:63-4.

16. Hao JH. Observation of effect of amlodipine and benazepril on two level prevention of hypertensive cerebral stroke. Chinese Community Doctors 2016;32:28-28, 30.

17. Liu CP, Hou YG, Zhu RY, et al. Therapeutic effects of levoamlodipine maleate on blood pressure of hypertensive patients after cerebral stroke. Hebei Yiyao 2018;40:3277-80.
18. Cao HZ, Wu LK, Lu WX. Effect of Nimodipine in the adjuvant treatment of cognitive impairment after cerebral infarction and its influence on cognitive function. Zhongguo Dangdai Yiyao 2019;26:98-100, 103.

19. Jiang $X$. Status quo and prospects of stroke prevention and its nursing care. Chinese Nursing Research 2017;31:7-12, 13.

20. Ly J, Maquet P. Clinical management of acute stroke. Rev Med Liege 2018;73:333-7.

21. Bridwell RE, Long B, April MD. Do Calcium Antagonists Decrease Mortality or Dependency in Acute Ischemic Stroke? Ann Emerg Med 2019;74:575-7.

(English Language Editor: K. Brown)
Cite this article as: Feng A, Wang W, Du C, He M. A systematic review and meta-analysis of early diagnosis and treatment of hypertensive stroke under calcium channel blockers. Ann Palliat Med 2021;10(6):6715-6725. doi: 10.21037/ apm-21-1272 